\title{
DEVELOPMENT AND APPLICATION OF ATM PROTOCOL CONFORMANCE TEST SYSTEM
}

\author{
Sungwon Kang, Youngsoo Seo, Deukyoon Kang, Mijeong Hong, \\ Junhwan Yang, Ilkook Koh, Jaehwi Shin, *Sangjo Yoo, **Myungchul Kim \\ Korea Telecom Telecommunications Network Laboratory \\ Junmin-dong 463-I, Yusung-gu, Taejon, 305-390, Korea \\ * Korea Advanced Institute of Science and Technology \\ Gusung-dong 373-1, Yusung-gu, Taejon, 305-701, Korea \\ ** Information and Communication University \\ Hwaam-dong 58-4, Yusung-gu, Taejon, 305-348, Korea \\ Email: \{kangsw, sys01, dykang, Ihdo, jhy, kok, jhshin \} @sava.kotel.co.kr \\ *sangjo@sdivision.kaist.ac.kr \\ **mckim@icu.ac.kr
}

\begin{abstract}
This paper presents development and application of ATM Conformance Test System (ACTS), an automated test system for ATM protocols. ACTS is a test system that checks conformance of ATM terminal and network equipment implementing either ITU-T or ATM Forum user-network interface. This paper, after presenting the methodology and process used for developing ACTS, conducts case studies of its applications to real ATM equipment. By applying ACTS, we were able to detect numerous problems in protocol implementations of ATM equipment and analyze causes of the problems, thereby demonstrating the efficacy of ACTS as an efficient automated testing tool. Furthermore, by predicting the potential effects of the problems on interoperability, we show how ACTS can be used as a useful tool for ensuring interoperable ATM equipment.
\end{abstract}

Keywords: ATM, Communication Protocol, Conformance Testing

\section{INTRODUCTION}

As the users demand faster and higher-volume multimedia telecommunication services, Asynchronous Transfer Mode (ATM) has been widely adopted 
as the base technology for Broadband-Integrated Services Digital Network (BISDN) services in many countries. Korea is not an exception and has started as a national project building a nation wide high-speed network based on ATM in 1993. A variety of equipment made by different vendors is expected to be deployed to construct the ATM network.

In order for communication equipment to interoperate, they should follow a common set of protocols. For this, ITU-T and ATM-Forum standardized ATM protocols. Therefore not only should ATM products follow the protocol standards but also it is essential to have efficient means to determine whether the protocol implementations indeed follow the standards correctly. The necessity of conformance test for ATM protocols as such means cannot be overemphasized because, in contrast with the case of PSTN, ATM networks rely on complicated protocols.

Most of the papers published so far about ATM protocols conformance test have focused on test generation methodologies $[1,2,3]$. On the other hand, only a few papers have been published on comprehensive ATM conformance test systems capable of testing for the complicated ATM protocol requirements of ITU-T and ATM Forum such as the ATM signaling protocol; e.g., [4] is about the design of an ATM conformance test system and [5] explains a commercial ATM test product. However, those systems generally could not keep up with the progress of standardization or meet the testing needs in a timely manner. Also conformance test system for the user-side ATM Forum UNI3.1 signaling protocol was not completely developed in those systems. Moreover, the published works on them were insufficient in showing the efficacy of their test systems by applying the systems and analyzing the application results for feedback.

In order to provide high-quality ATM protocol conformance tests without the limitations mentioned earlier, we started to develop ATM Protocol Conformance Test System (ACTS) in 1996. ACTS is a test system that determines conformance of ATM equipment against ATM UNI standards of either ITU-T or ATM Forum. For two years since then, ACTS has grown up so as to cover most of the major ATM UNI protocols including the user-side and network-side signaling protocol. In this paper, we discuss the development of ACTS and its application.

The rest of the paper is organized as follows; Section 2 surveys Signaling layer, SAAL layer, and ATM layer of ATM UNI protocol; test architectures for the layers are described in Section 3; in Section 4, we introduce the procedures and methods for developing Abstract Test Suite (ATS) and Executable Test Suite (ETS) and explain ACTS in detail; Section 5 conducts case studies of ACTS application and discusses expected interoperability problems based on the application results; finally, Section 6 concludes by explaining the future plan for ACTS development and further applications. 


\section{ATM PROTOCOL STACK}

ATM protocols are standardized both in ITU-T [6, 7, 8] and ATM Forum [9]. The ATM protocol stack can be functionally divided into the user plane, the control plane, and the management plane. ACTS focuses on the control plane shown as the shaded part of Figure 1.

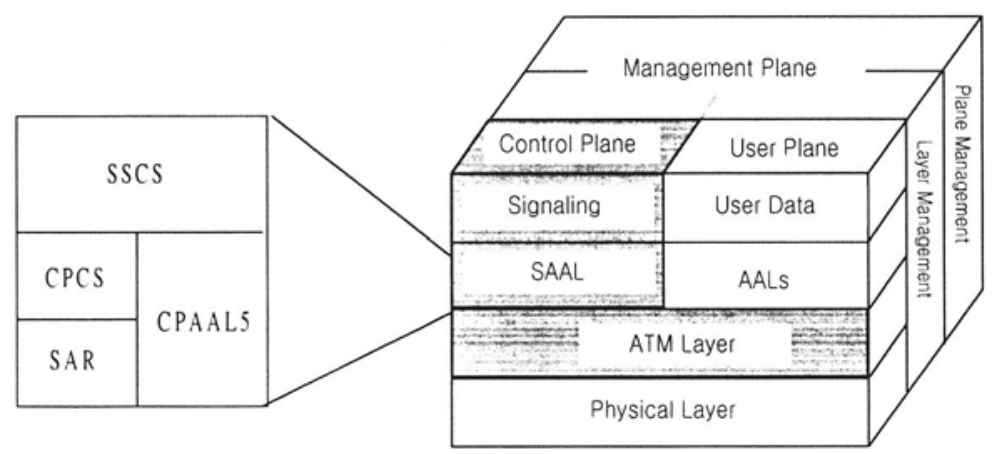

AAL: ATM Adaptation Layer

SSCS: Service Specific Convergence Sublayer

SAR: Segmentation And Reassembly
SAAL: Signaling AAL

CPCS: Common Part Convergence Sublayer

CPAAL5: Common Part AAL5

Figure I The structure of the ATM protocol stack

The signaling protocol specifies the formats of the signaling messages and the procedures to dynamically establish, maintain and release connections at UNI and to handle error cases.

The payload of ATM layer has a fixed size of 48 octets so that most of the higher layer services need to adapt variable length packets to the payload. This adaptation is provided by AAL. There are four AAL classes depending on applications: AAL1, AAL2, AAL3/4, and AAL5. The signaling protocol uses AAL5.

The ATM layer provides functions such as cell switching and cell multiplexing/demultiplexing between the AAL and the physical layer.

\section{DECIDING ON TEST ARCHITECTURES}

ATS is a prerequisite of conformance test system development. In order to develop ATS we first have to decide on the test coverage, the test method and the test architecture. ISO9646 proposes the conformance test methods: the Local test method, the Distributed test method, the Coordinated test method, and the Remote test method [10]. The three test methods except the Remote 


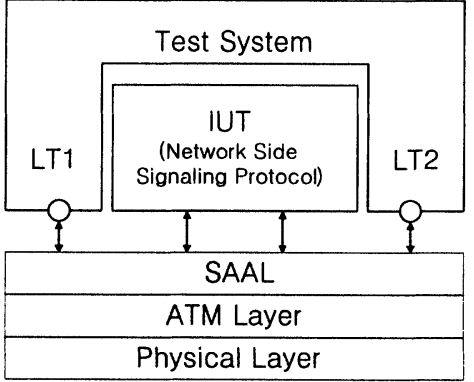

$\mathrm{O}: \mathrm{PCO}$

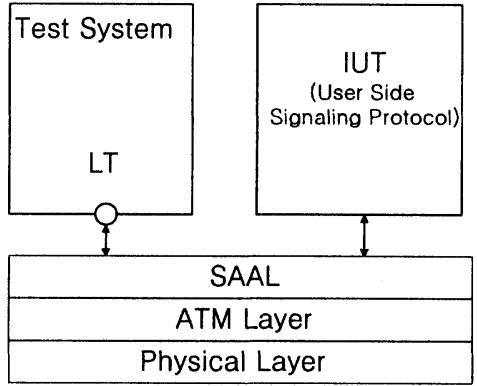

(b) For a terminal

(a) For a switch

Figure 2 Test architectures for the signaling protocol

test method need an Upper Tester (UT) and a Lower Tester (LT). In the Local test method, the test system and an Implementation Under Test (IUT) reside in the same system and, in the Distributed test method, the test coordination procedure is required between UT and LT. The coordinated test method uses Test Management Protocol (TMP) for test coordination. These three methods pose difficulties in testing by the third party because they need to access both the upper and the lower interfaces.

On the other hand, the Remote test method is suitable for the third party testing because it does not require UTs, although it tends to give lower test coverage than the others owing to absence of UTs. For these reasons, we

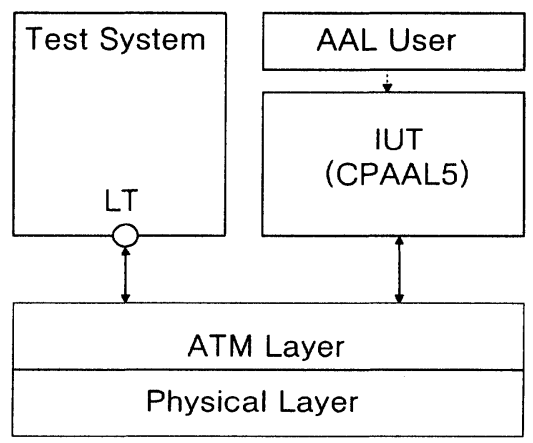

Figure 3 Test architecture for CPAAL5 protocol 
adopted the Remote test method as the test method for each layer of the ATM protocols.

In order to test a switch or a terminal implementing the UNI signaling protocol, we selected the test architectures in Figure 2(a) and in Figure 2(b), respectively. In Figure 2(a), incoming and outgoing calls can be tested using two Points of Control and Observation (PCOs), LT1 and LT2.

SAAL is divided into two sublayers CPAAL5 and SSCS as shown in Figure 1. ACTS aims to test only CPAAL5, which is independent of the upper layer, and for that we choose the test architecture in Figure 3.

ATM layer implementations can be either in an Intermediate System (IS) or in an End System (ES). Figure 4(a) shows the test architecture for an IS and Figure 4(b) for an ES. To test for an IS, it takes two PCOs because it relays cells between two interfaces.

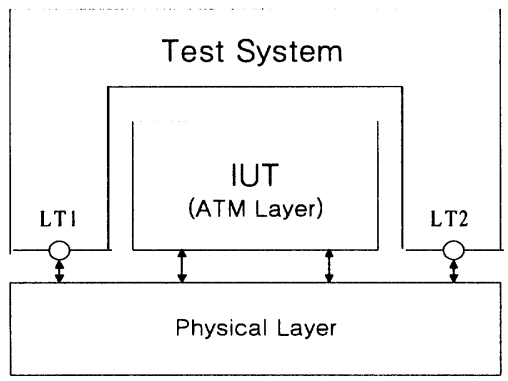

(a) For an intermediate system

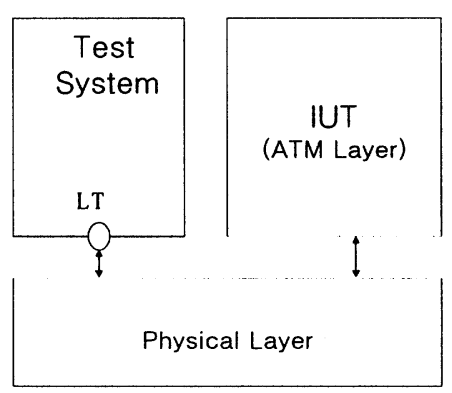

(b) For an end system

Figure 4 Test architectures for the ATM layer protocol

\section{DEVELOPMENT OF THE TEST SYSTEM}

In this section, we describe the test system development process carried out after the test architecture selection of Section 3 that reflects the characteristics of each layer. Section 4.1 explains the process of system development. Sections 4.2 and 4.3 describe respectively development of ATS and development of executable test software based on it.

\subsection{METHOD AND PROCEDURE OF TEST SYSTEM DEVELOPMENT}

In order to derive ATS from a protocol specification, we used test matrix, which is basically a tabular representation of a protocol Finite State Machine(FSM), as the intermediate step for deriving test cases. Test matrix utilizes 
both formal and informal description for each state of what message is produced and what action is taken by the protocol entity, from which all the test purposes are derived, which in turn are the bases for deriving individual test scenarios. Following such steps test system development proceeded as shown in Figure 5 ; 1) write ATS using a TTCN editor from test matrix and convert it to Machine Processible (MP) file, 2) generate executable test software in C program using a TTCN-to-C translator, 3) develop C program support code, 4) generate the platform-dependent executable test software by compiling and linking the results of steps 2) and 3).

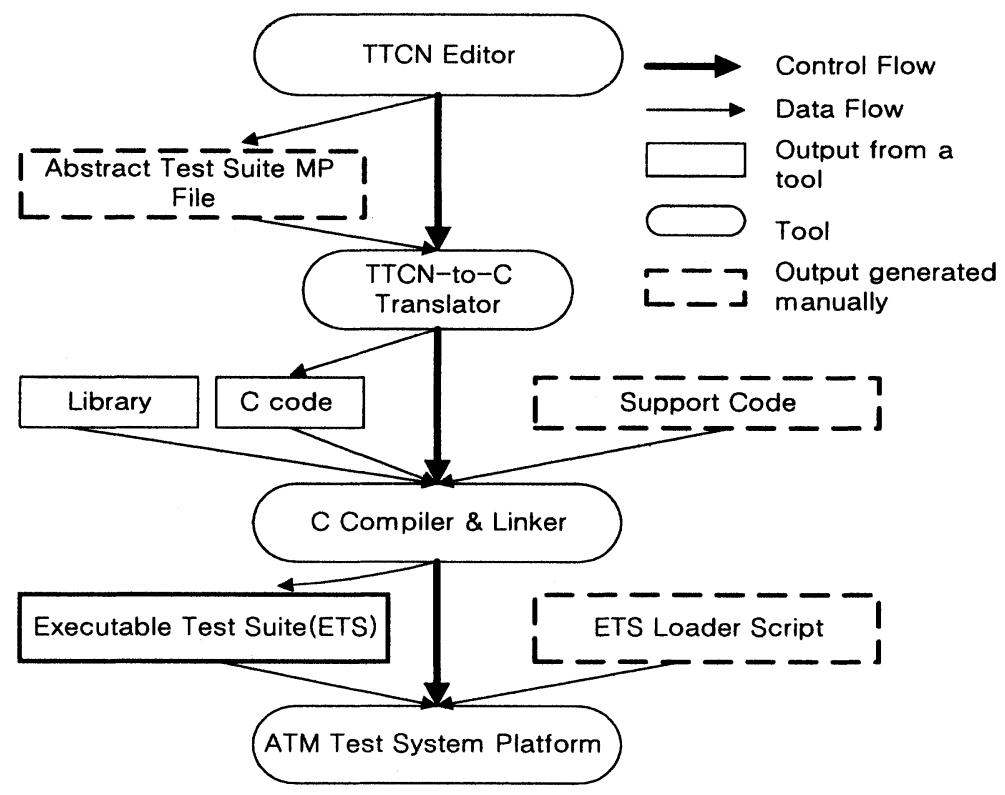

Figure 5 Flowchart for test system development procedure

\subsection{DEVELOPMENT OF ATS}

Protocol specification usually includes options and parameters in addition to mandatory requirements. Implementers may choose to implement or not to implement options and need to select values for parameters for the particular implementation. Questionnaire to identify options and parameters is called Protocol Implementation Conformance Statement (PICS). Questionnaire for the items not included in PICS but necessary for testing is called Protocol Implementation eXtra Information for Testing (PIXIT).

In this study, ATS was developed so that test cases can be automatically selected and parameter values can be conveniently substituted depending on 
the PICS and PIXIT contents for the particular implementations. Our ATSs can be easily converted by a TTCN-to-C translator to executable test software that can be used on any test platform when combined with suitable platform dependent programs.

Once test purposes and test methods are determined, the next step is to develop ATS, which describes a test scenario for each test purpose. One may consider a natural language for such description but we chose Tree and Tabular Combined Notation (TTCN) [10], a formal test description language standardized as ISO9646. The reasons are; 1) TTCN is an international standard language for describing communication protocol test scenarios, 2) By using a formal language, we can take advantage of automatic syntax checking using the TTCN tools and avoid possible vagueness and ambiguities in test scenario description, 3) Using automatic or semi- automatic program generation tools, efficient development of executable test systems is possible.

ATS for the UNI3.1 user-side signaling protocol was developed from scratch referring to ATM Forum ATS [11]. ATS for the network-side signaling protocol was developed, based on UNI 3.1 network-side signaling ATS [12], which is still under development in ATM Forum, by analyzing in detail the test purposes, the declarations, the constraints and the dynamic behaviors and correcting problems due to misinterpretation of the specification or errors in the test scenario descriptions. There was no ITU-T Q.2931 ATS known to us and it was developed by first comparing ITU-T Q.2931 and ATM Forum UNI 3.1 and then identifying differences between them.

ATS for CPAAL5 was developed in the situation that a similar or related ATS did not exist. CPCS was the main target in CPAAL5 testing and in order to access Cell Loss Priority and ATM User-to-User indication in ATM Cell Header Payload Type we directly looked into ATM cells instead of using the ATM primitives. ${ }^{1}$

For ATM layer ATS we used ATM Forum ATM Layer conformance test suite $[14,15]$ and since there is no ITU-T I.361 ATS we reused most of the test cases of ATM Forum ATSs and added test cases for Cell Rate Decoupling, Generic Flow Control, Operation and Maintenance and deleted or modified other test cases.

\subsection{DEVELOPMENT OF EXECUTABLE TEST SOFTWARE}

In order to derive ETS from ATS, in the case of the signaling protocol and the ATM layer we applied a TTCN-to-C translator to MP form ATS generated

\footnotetext{
'The ATS developed in this study was adopted by ATM Forum as the ATM Forum CPAAL5 Abstract Conformance Test Suite [13].
} 
from a TTCN editor to obtain C program [5]. Sending and receiving PDUs depends on the lower layer and therefore cannot be automatically generated by a TTCN-to-C translator. The part not automatically generated should be manually programmed and we call such programs support codes.

\subsubsection{Development of Executable Test Software for the Signaling Pro-} tocol. Sending and receiving PDUs of the signaling protocol was implemented using SAAL API supplied by ATM test platform [5]. The support code for PDU sending and receiving that we developed has the structure shown in Figure 6.

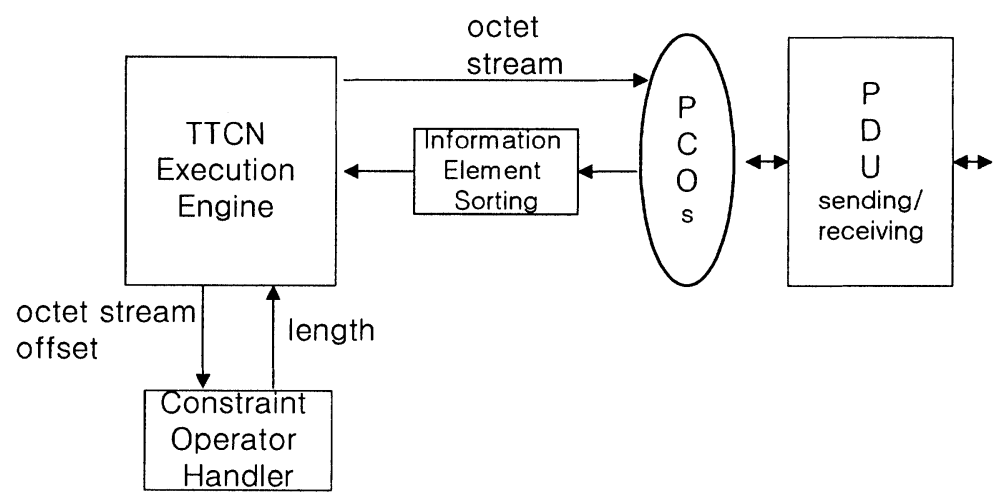

Figure 6 Structure of executable test software for the ATM signaling protocol

In Figure 6, the TTCN execution engine generated by the translator is essentially in charge of executing TTCN dynamic description. The TTCN execution engine sends and receives PDUs through PCOs, compares and calculates PDU constraints and received PDUs. When encountering constraint operators such as ANY and ANYOROMIT, it calls constraint operator handling routines with offset values indicating the location of the operators in the PDUs. Constraint operator handling routines analyze existence and the lengths of Information Elements (IEs) based on the octet string of PDU and offset values and return the results to the TTCN execution engine. Program codes for the operations ANY, ANYOROMIT and IF_PRESENT were realized so that they can be generally applied to IEs as units. When certain fields of IEs have such operations, special program codes were prepared for them.

PDUs of the signaling protocol are specified such that optional IEs can appear in any order. In order to make executable test software handle this, we included in support code a sorting routine that rearrange IEs in a predetermined order. 
Most of the developed support code was commonly used both for the userside and the network-side signaling test system and when discrepancy exists slight modifications sufficed.

4.3.2 Development of CPAAL5 Executable Test Software. In the case of CPAAL5, we developed the whole test system by manual programming. CPAAL5 executable test software consists of 1) executable programs for test management module, 2) test library that supports easy programming of test cases described in TTCN and 3) executable test cases. Configuration of CPAAL5 test system is depicted in Figure 7.

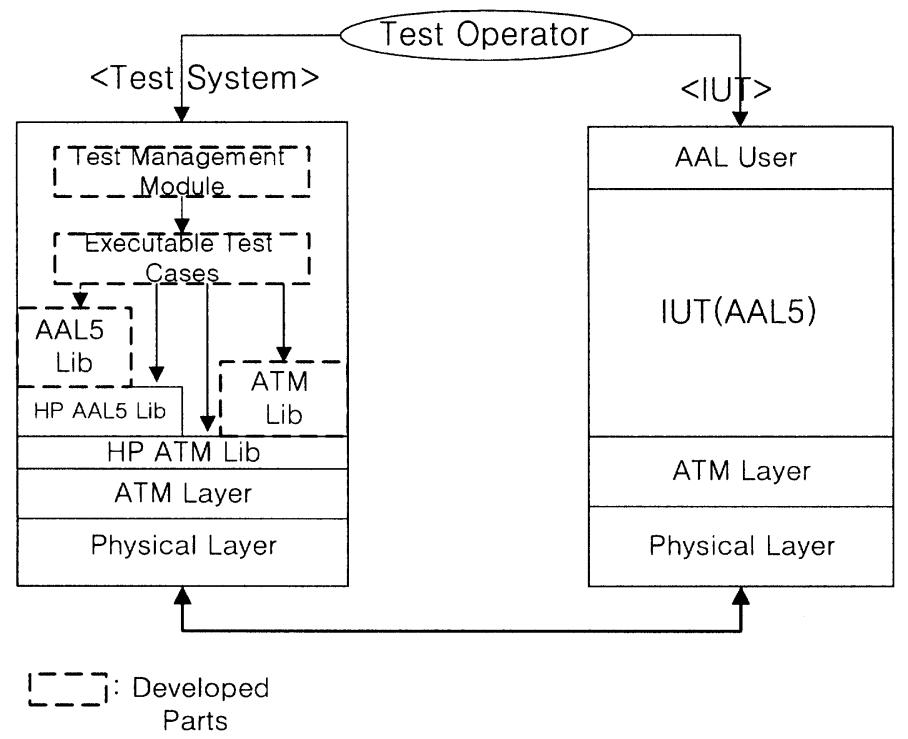

Figure 7 Configuration of CPAAL5 test system

In order to avoid dependency on test system platform, we developed test libraries for the functions : 1) initializing test modules, 2) setting of filter that only captures ATM cells with desired VPI/VCI, 3) to store ATM cells in capture buffer, 4) making up AAL5 PDUs by reassembling cells in the capture buffer, 5) reproducing the initial cell in the capture buffer and 6) reproducing cells sequentially. Test cases generally have the following functions: 1) setting of the capture filter, 2) setting of constraints, 3) receiving cells in a buffer, 4) reassembling cells into AAL5 PDUs, 5) comparing PDUs against predetermined constraints and 6) giving verdicts. 


\subsubsection{Development of Executable Test Software for the ATM Layer.} In order to load executable test software for the ATM layer on test system platform, there are certain prerequisites. For example, we must handle lengths and values of test suite parameters, information about the physical module and information about options. In general, the following cannot be generated by the translator and must be manually programmed: tester initialization, library for lower layer protocols and interface, initialization after execution of the individual test cases or the test suite and informally described test suite operations. We programmed support codes for them to get the final executable test software. The test software developed by the methods of Section 3 and 4 has been loaded on the ATM test system platform as shown in Table 1 and currently is in operation for conformance testing of ATM equipment.

Table 1 Specification of the ATM test system and system platform

\begin{tabular}{|c|c|c|c|c|}
\hline \multirow{7}{*}{$\begin{array}{l}\text { Test } \\
\text { System }\end{array}$} & \multirow{2}{*}{\multicolumn{2}{|c|}{ Protocol }} & \multicolumn{2}{|c|}{ Number of Test Cases } \\
\hline & & & ITU-T & ATM Forum \\
\hline & \multirow{2}{*}{$\begin{array}{c}\text { Signaling } \\
\text { Protocol }\end{array}$} & User-side & 517 & 581 \\
\hline & & Network-side & 583 & 661 \\
\hline & \multicolumn{2}{|c|}{ CPAAL5 } & 17 & 17 \\
\hline & \multirow[t]{2}{*}{$\begin{array}{l}\text { ATM } \\
\text { Layer }\end{array}$} & $\begin{array}{l}\text { Intermediate } \\
\text { System }\end{array}$ & 49 & 47 \\
\hline & & End System & 40 & 37 \\
\hline \multirow{3}{*}{$\begin{array}{l}\text { System } \\
\text { Platform }\end{array}$} & \multicolumn{2}{|c|}{ OS } & \multicolumn{2}{|l|}{ HP UX 9.02} \\
\hline & \multicolumn{2}{|c|}{ Line Interface } & \multicolumn{2}{|c|}{ 155Mb/s Optical (SONET/SDH) 2 Module } \\
\hline & \multicolumn{2}{|c|}{ ATM Cell Processor } & \multicolumn{2}{|c|}{$\begin{array}{l}\text { ATM Cell and AAL/UNI } \\
\text { Signaling Protocol Processor } 2 \text { Module }\end{array}$} \\
\hline
\end{tabular}

\section{APPLICATIONS OF ACTS}

Generally, we follow the procedure described in Figure 8 in order to perform protocol conformance test. First, we decide if the IUT conforms to its specification by examining the IUT's PICS. This step is called static conformance test. If the IUT passes the test, a set of executable test cases is selected and their parameters are set to proper values according to the IUT's PICS and PIXIT. Then with the selected instantiated executable test cases, we test the IUT. This step is called dynamic conformance test.

In this section, we present case studies of dynamic conformance test by applying ACTS to ATM equipment, focusing mainly on the signaling protocol. 


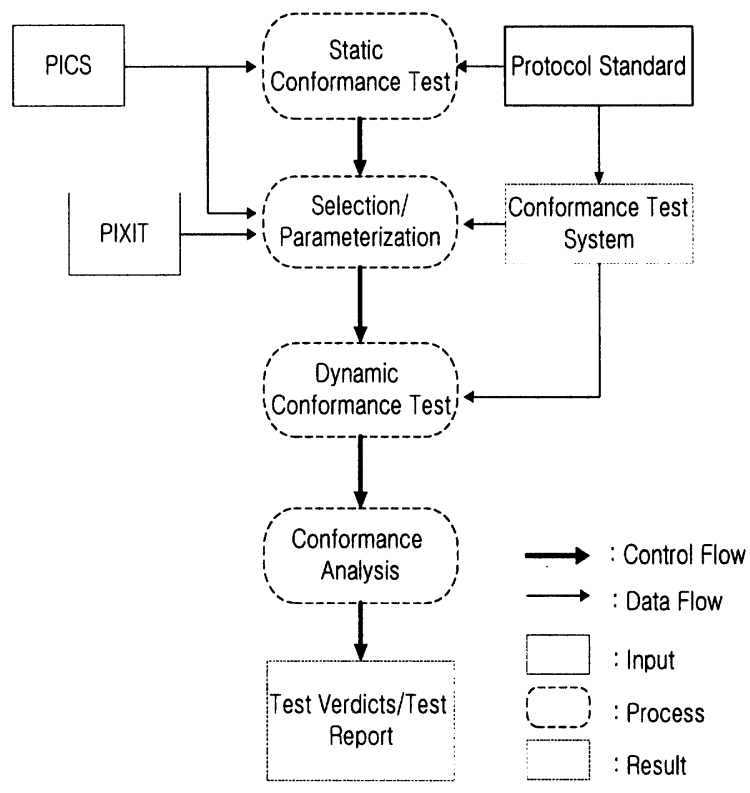

Figure 8 Procedure for protocol conformance testing

\subsection{CASE STUDY I: CONFORMANCE TESTING OF ATM TERMINALS}

In this conformance testing of two commercial ATM terminals $\mathrm{Ht}$ and $\mathrm{Ft}$ from vendors $\mathrm{H}$ and $\mathrm{F}$, respectively, test cases for the user-side signaling protocol were selected in accordance with the individual features of the equipment. In both tests, there were fail and inconclusive verdicts.

The two terminals received fail verdicts for all the test cases of the incoming call test group. According to the specification [9], Broadband Low Layer Information (BLLI) and AAL Parameter (AALP) IEs are optional. Thus, the test cases of the incoming call test group were designed not to include those IEs in SETUP messages. However, the IUTs rejected SETUP by RELEASE COMPLETE. It was found out that $H t$ requires BLLI as mandatory and $F t$ requires both BLLI and AALP. Consequently, test cases of the call release test group using an incoming call received either fail or inconclusive verdicts.

\subsection{CASE STUDY II: CONFORMANCE TESTING OF AN ATM SWITCH}

In this conformance testing of the network-side signaling protocol, test cases were selected and applied to an ATM switch $F s$ from a vendor F. There were fail 
verdicts in the call release test group because of state error, message sequence error, and IE content error.

The state error occurs when the state of an IUT, i.e. the state of the signaling entity of a switch, is different from the expected state. According to [9], after sending RELEASE the state of the signaling entity should be $N 12$ but it became NO.

The message sequence error occurs when an IUT in a specific state sends a message different from what is expected. According to [9], after an IUT sends CALL PROCEEDING, if it cannot process a call for some reason, it should send RELEASE but $F s$ sent RELEASE COMPLETE.

The IE content error occurs when an IUT sends a message with incorrect IE based on the IEs of the received message. Fs relayed RELEASE with incorrect CAUSE IE based on the RELEASE message received from a calling party.

\subsection{CASE STUDY III: CONFORMANCE TESTING OF AN ATM NT}

In this conformance testing of an ATM Network Termination (NT) En from a vendor E, test cases of the network-side signaling protocol were selected and applied in accordance with its features. There were fail verdicts and their major causes are as follows; 1) En processed a call without inspecting the mandatory IEs of a received message; 2) En did not implement the restart procedure and its relevant messages; 3 ) En did not perform a proper VPI/VCI negotiation procedure and 4) there existed an error in the call release function upon receiving an incorrect signaling message.

\subsection{EXPECTED INTEROPERABILITY PROBLEMS AMONG ATM EQUIPMENT}

In this section, we discuss possible interoperability problems between a terminal and a switch and between two terminals based on conformance test results.

In this paper, interoperability means the capability of two or more systems that when they are interconnected they together perform given functions as desired. Note that even when each system conforms to a relevant specification individually, they might not be interoperable or vice versa because of incompleteness of specifications and/or conformance test. However, interoperability can be significantly improved by detecting possible interoperability problems and by fixing them in advance based on the results of conformance testing. Let us illustrate it with the following three cases:

1) Expected interoperability problems between $\mathrm{Ht}$ and $\mathrm{Fs}$

Through conformance testing of $F s$, we concluded that it had no conformance problem except some minor ones in the call release function. In other words, it 
successfully relayed BLLI and AALP IEs from the calling party to the called party as well as other IEs in the SETUP message. Thus, the incoming call may be established without problems as well as the outgoing call although there might exist minor turbulence in the call release.

2) Expected interoperability problems between $\mathrm{Ht}$ and $\mathrm{Ft}$

As explained in Section 5.1, both $H t$ and $F t$ failed some test cases because they did not follow the specification in their selection of mandatory or optional information. For instance, they required BLLI as mandatory in an incoming SETUP message even though they are optional; this violation may result into severe interoperability problems with other ATM equipment.

Even though $H t$ and $F t$ required BLLI as mandatory, $H t$ additionally required AALP IE in an incoming SETUP message as mandatory. Thus calls originated from $F t$ could be rejected by $H t$.

3) Expected interoperability problems between terminals and $E n$

En has a problem with VPI/VCI negotiation; there is the possibility that two different VPI/VCIs are set up for a single call so that they might remain dangling even after the call release. Also $H t$ and $F t$ can request $E n$ to restart the indicated virtual channels or all virtual channels controlled by $E n$. But $E n$ lacked this feature. Hence, a SETUP message from $E n$ included incorrect contents. Thus some application services on the terminal using signaling information may not work.

The above analyses are summarized in Table 2. In the table $A-B$ means that $A$ and $B$ are directly connected with each other.

Table 2 Expected degrees of interoperability between IUTs

\begin{tabular}{|l|c|l|}
\hline Configuration & $\begin{array}{c}\text { Expected } \\
\text { Interoperability }\end{array}$ & \multicolumn{1}{|c|}{ Reason } \\
\hline 1. Ht $-F t$ & Low & $\begin{array}{l}\text { A call initiated by } H t \text { will be rejected by } F t \text { if } H t \text { does } \\
\text { not send SETUP with AALP }\end{array}$ \\
\hline 2. Ht $-F s$ & High & No significant conformance problems discovered \\
\hline 3. Ht $-E n$ & Medium & $\begin{array}{l}\text { Established calls as well as establishing calls may be } \\
\text { interrupted or disconnected due to possible incorrect } \\
\text { VPI/VCI negotiation of } E n\end{array}$ \\
\hline 4. Ft $-E n$ & Medium & Same as the configuration 3. $H t-E n$ \\
\hline
\end{tabular}

\section{CONCLUSION}

In this paper, we have described the motivation behind developing ACTS, the development process and its applications. ACTS was developed so that ATM conformance tests are timely available as the testing needs arise and can 
keep up with change and progress of standardization. We applied ACTS to testing of various ATM equipment and analyzed the test results and discussed possible interoperability problems based on the results of conformance testing.

Through applications of ACTS, we observed that the ATM protocol implementations, especially those for the signaling protocol, had many problems. If we consider that these equipment probably have gone through many stages of testing during the development process before submitted for testing with ACTS, we may conclude that ACTS has a fairly good test coverage.

ACTS is used in Korea Telecom, the major carrier of Korea, for decision making related to the procurement of ATM equipment and can be used during the development stage as a test tool or during the operation stage as an analysis tool to track down problems. It can also be used to grant certification to vendors for standards conformance.

Currently, ACTS can perform conformance test for ATM layer, CPAAL5 layer, UNI 3.1 signaling protocol, and Q.2931. Now its scope is being extended to include signaling protocols of Network Node Interface (NNI). Since the ultimate goal of protocols is in achieving interoperation of communication services, the ability to verify protocol interoperability in a direct manner is an extremely important sort of testing. Recognizing such importance, we are developing an interoperability test system for Q.2931 and UNI 3.1 signaling protocols. Further augmented with a performance test system, ACTS is evolving into an integrated ATM test system which can perform conformance, interoperability, and performance test on a common system platform.

\section{References}

[1] Bechtold, R., Gattung, G., Henniger, O., Paule, C.,"Test case generation for ATM protocols using high-level Petri net models",IFIP TC6/WG6.1 The 9th International Workshop on Testing of Communication Systems '(IWTCS'96), Darmstadt, Germany, 1996.

[2] Vandevenne,L., Sutter, D., Goossens, E., "Testing the broadband network: a quickly shifting demand for test equipment solutions", IFIP TC6/WG6.1 The 9th International Workshop on Testing of Communication Systems (IWTCS'96), Darmstadt, Germany, 1996.

[3] Witteman, M. F., Wuijtswinkel, R. C., "ATM braodband network testing using the ferry principle", IFIP TC6/WG6.1 The 9th International Workshop on Protocol Test Systems (IWPTS'93), Pau, France, 1993.

[4] Kim, K., Kim W., Hong, B., "Experiences with the design of B-ISDN Integrated System", IFIP TC6/WG6.1 The 8th International Workshop on Protocol Test Systems (IWPTS'95), Evry, France, September 1995.

[5] Hewlett Packard BSTS Manual, 1995-1996. 
[6] ITU-T Recommendation I.361, "B-ISDN ATM Layer Specification", 1995.

[7] ITU-T Recommendation I.363, "B-ISDN ATM Adaptation Layer Specification", December 1993.

[8] ITU-T, "ITU-T draft Recommendation Q.2931: B-ISDN User-Network Interface Layer 3 Specification for Basic Call/Bearer Control", March 1994.

[9] ATM Forum, "ATM User-Network Interface Specification", Version 3.1, 1994.

[10] ISO/IEC, "ISO/IEC IS 9646 Part 1-7, Information Technology - Open Systems Interconnection - Conformance Testing Methodology and Framework", 1995.

[11] Collica, L., Yoo, S. J., Kang, S.,"96-0979: ATS for the UNI 3.1 Signaling -User-side", BTD- TEST-ATS-sig31user-01.00, 1996.7.

[12] ATM Forum, " Conformance Abstract Test Suite for Signalling (UNI 3.1) for the Network Side", af-test-0090, 1997.9.

[13] ATM Forum, "Conformance Abstract Test Suite for the ATM Adaptation Layer (AAL) Type 5 Common Part", af-test-0052, 1996.3.

[14] ATM-Forum, "ATM Layer Conformance Abstract Test Suite for Intermediate Systems", af- test-0030, 1995.4.

[15] ATM-Forum, "ATM Layer Conformance Abstract Test Suite for End Systems", af-test-0041, 1995.7.

[16] Burmeister, J., Rennoch, A., "Application of a LOTOS based Test Environment on AAL5", IFIP TC6/WG6.1 The 8th International Workshop on Protocol Test Systems (IWPTS'95), Evry, France, September 1995.

[17] Grabowski, J. Scheurer, R. Dai, Z. R., Hogrefe, D., "Applying SaMsTaG to the B-ISDN protocol SSCOP", IFIP TC6/WG6.1 The 10th International Workshop on Testing of Communication Systems (IWTCS'97), Cheju Island, Korea, September 1997.

Sungwon Kang received a B.A. from Seoul National University in Korea in 1982 and a M.S. and a Ph.D. in computer science from the University of Iowa in U.S.A in 1989 and 1992. In 1993, he joined Korea Telecom R\&D Group and has been Head of Network Testing Department since 1997. From 1995 to 1996, he was a guest researcher at National Institute of Standards and Technology in U.S.A. In 1997, he served as the co-chair of the 10th International Workshop on Testing of Communicating Systems. His areas of interest include communication networks and communication protocol testing.

Youngsoo Seo received a B.S. and a M.S. in industrial engineering from Hanyang University in 1991 and 1993, respectively. Currently he is with Korea Telecom R\&D Group as a member of technical staff. His research interests include protocol engineering and network engineering. 
Deukyoon Kang has been working as a member of technical staff in Korea Telecom R\&D Group since 1995. He has participated in projects such as ATM Signaling Protocol Validation and ATM Conformance Test S/W development. Currently, he is participating in the design of MPLS Test Architecture.

Mijeong Hong received a B.S. in electronic engineering from Kyungbuk University in 1990. Currently she is with Korea Telecom R\&D Group as a member of technical staff.

Junhwan Yang received the B.S. and M.S. degrees in electronic engineering from Kon-kuk University in 1989 and 1991,respectively. Currently he is with the Korea Telecom R\&D Group as a member of technical staff. His research interests include telecomunication/data network protocols, testing of telecommination/data network systems and developing test systems.

Ilkook Koh received B.S. in computer science from Chosun University in 1995. currentl he is with the Korea Telecom R\&D Group as a member of technical staff. His research interests include protocol conformance testing on ATM/B-ISDN.

Jaehwi Shin received a B.S. and a M.S. in electronic engineering from Hanyang University and Seoul National University in 1991 and 1993, respectively. Currently he is with Korea Telecom R\&D Group as a member of technical staff. His research interests include intelligent networks, communication protocol testing, digital signal processing and circuit theory.

Sangjo Yoo received a B.A. in electric communication engineering from Hanyang University in 1988 and M.S. in electrical engineering from the Korea Advanced Institute of Science and Technology in 1990. Currently he is with the Korea Telecom R\&D Group as a member of technical staff.

Myungchul Kim received B.A. in Electronics Engineering from Ajou University in 1982, M.S. in Computer Science from the Korea Advanced Institute of Science and Technology in 1984, and $\mathrm{Ph}$. D in Computer Science from the University of British Columbia, Vancouver, Canada, in 1993. Currently he is with the faculty of the Information and Communications University, Taejon, Korea. Before joining the university, he was a managing director in Korea Telecom R\&D Group for 1984 - 1997 where he was in charge of research and development of protocol and QoS testing on ATM/B-ISDN, IN, PCS and Internet. He has also served as a member of Program Committees for IFIP International Workshop on Testing of Communicating Systems, IEEE International Conference on Distributed Computing Systems, and IFIP International Conference on Formal Description Technique / Protocol Specification, Testing and Verification, the chairman of Profile Test Specifications - Special Interest Group of Asia-Oceania Workshop (for 1994 1997), and co-chair of the 10th IWTCS'97. His research interests include Internet, protocol engineering, telecommunications, and mobile computing. 